\title{
Retinopathy, Neuropathy, and Subsequent Cardiovascular Events in Patients with Type 2 Diabetes and Acute Coronary Syndrome in the ELIXA: The Importance of Disease Duration
}

\author{
Jelena P. Seferovic $\mathbb{D}^{1}{ }^{1}$ Rhonda Bentley-Lewis, ${ }^{2}$ Brian Claggett, ${ }^{1}$ Rafael Diaz, ${ }^{3}$ \\ Hertzel C. Gerstein, ${ }^{4}$ Lars V. Køber, ${ }^{5}$ Francesca C. Lawson, ${ }^{6}$ Eldrin F. Lewis, ${ }^{1}$ \\ Aldo P. Maggioni, ${ }^{7}$ John J. V. McMurray, ${ }^{8}$ Jeffrey L. Probstfield, ${ }^{9}$ Matthew C. Riddle, ${ }^{10}$ \\ Scott D. Solomon, ${ }^{1}$ Jean-Claude Tardif, ${ }^{11}$ and Marc A. Pfeffer ${ }^{1}$ \\ ${ }^{1}$ Cardiovascular Division, Brigham and Women's Hospital, Harvard Medical School, Boston, MA, USA \\ ${ }^{2}$ Massachusetts General Hospital, Harvard Medical School, Boston, MA, USA \\ ${ }^{3}$ Estudios Clínicos Latinoamérica, Rosario, Argentina \\ ${ }^{4}$ McMaster University, Hamilton, ON, Canada \\ ${ }^{5}$ Rigshospitalet Copenhagen University Hospital, Copenhagen, Denmark \\ ${ }^{6}$ Sanofi U.S., Bridgewater, NJ, USA \\ ${ }^{7}$ Research Center of the Italian Association of Hospital Cardiologists, Florence, Italy \\ ${ }^{8}$ British Heart Foundation Cardiovascular Research Centre, University of Glasgow, Glasgow, UK \\ ${ }^{9}$ University of Washington Medical Center, Seattle, WA, USA \\ ${ }^{10}$ Oregon Health and Science University, Portland, OR, USA \\ ${ }^{11}$ Montreal Heart Institute, Université de Montréal, Montreal, Canada
}

Correspondence should be addressed to Jelena P. Seferovic; jpseferovic@gmail.com

Received 25 March 2018; Accepted 18 September 2018; Published 16 December 2018

Guest Editor: João R. de Sá

Copyright (c) 2018 Jelena P. Seferovic et al. This is an open access article distributed under the Creative Commons Attribution License, which permits unrestricted use, distribution, and reproduction in any medium, provided the original work is properly cited.

Introduction. We investigated the association of diabetic retinopathy and neuropathy with increased risk of recurrent cardiovascular (CV) events in 6068 patients with type 2 diabetes mellitus (T2DM) and recent acute coronary syndrome (ACS) enrolled in the Evaluation of Lixisenatide in Acute Coronary Syndrome (ELIXA). Methods. History of retinopathy and neuropathy as well as duration of T2DM were self-reported at screening. Proportional hazards regression models were used to assess relationships between retinopathy, neuropathy, and recurrent CV events. Results. At screening, retinopathy and neuropathy were reported in $10.7 \%$ and $17.5 \%$ of patients, respectively, while $5.7 \%$ reported both. When adjusted for randomized treatment only, both retinopathy and neuropathy were associated with a primary composite outcome (CV death, nonfatal MI, stroke, or hospitalization for unstable angina) (retinopathy: HR 1.44, 95\% CI 1.19-1.75; neuropathy: HR 1.33, 95\% CI 1.12-1.57), CV composite (CV death, nonfatal MI, stroke, hospitalization for heart failure (HF)) (retinopathy: HR 1.57, 95\% CI 1.31-1.88; neuropathy: HR 1.38, 95\% CI 1.19-1.62), myocardial infarction (retinopathy: HR 1.38, 95\% CI 1.08-1.76; neuropathy: HR 1.26, 95\% CI 1.02-1.54), HF hospitalization (retinopathy: HR 2.03, 95\% CI 1.48-2.78; neuropathy: HR 1.71, 95\% CI 1.30-2.27), and all-cause mortality (retinopathy: HR 1.65, 95\% CI 1.28-2.12; neuropathy: HR 1.43, 95\% CI 1.14-1.78). When included in the same model, and adjusted for T2DM duration, there were no independent associations of either with CV outcomes, while T2DM duration remained strongly associated with all outcomes. Addition of demographic characteristics and $\mathrm{CV}$ risk factors did not further alter these relationships. Conclusions. In patients with T2DM and recent ACS, a history of retinopathy and/or neuropathy and longer T2DM duration could be considered clinical markers for high risk of recurrent $\mathrm{CV}$ events. This trial is registered with the ELIXA (Evaluation of Lixisenatide in Acute Coronary Syndrome), ClinicalTrials.gov registration number NCT01147250. 


\section{Introduction}

Cardiovascular (CV) risk in type 2 diabetes mellitus (T2DM) was associated with disease duration and severity of hyperglycemia in many studies [1-3]. In the Framingham Heart Study, duration of T2DM significantly and positively related to the risk of coronary heart disease mortality, but not morbidity, or CV disease (CVD) morbidity or mortality [2]. The Action in Diabetes and Vascular Disease: Preterax and Diamicron Modified Release Controlled Evaluation (ADVANCE) trial, on the contrary, showed that T2DM duration was independently associated with the risk of macrovascular complications and death [3]. In a metaanalysis of five trials, intensive glycemic control led to a reduction of nonfatal myocardial infarction (MI) incidence by one-sixth, with no significant effect on the incidence of nonfatal stroke, and both CV or all-cause mortality [4].

Additionally, duration of T2DM and severity of hyperglycemia are strong and consistent risk factors for the development and progression of microvascular diabetic complications-retinopathy (DR), neuropathy (DN), and nephropathy [5-8]. For example, in the very large and diverse T2DM population in ADVANCE, T2DM duration was independently associated with the risk of DR and nephropathy [3]. In the Maastricht Study, a T2DM-enriched population-based cohort study prediabetes, T2DM, and measures of hyperglycemia were independently associated with DR and DN [9]. Improved metabolic control was associated with $25 \%$ risk reduction of microvascular composite endpoint in the United Kingdom Prospective Diabetes Study (UKPDS) [10]. Similar effect has been shown for individual complications, DR $[5,11], \mathrm{DN}[6,11]$, and nephropathy $[7,11,12]$.

The association of diabetic nephropathy and increased risk of CVD has been revealed in multiple patient cohorts. Data from United States community-based study demonstrated that individuals with diabetic nephropathy were at four- and threefold higher risk for CV death and all-cause death, respectively, compared to those without [13]. In the ADVANCE study, both albuminuria and reduced estimated glomerular filtration rate (eGFR) were independently and continuously associated with the risk for CV and kidney outcomes in T2DM patients [14]. Similarly, among patients with type 1 diabetes followed for over 30 years, the development of diabetic nephropathy was associated with higher risks of CVD and renal events. However, this effect was almost entirely eliminated by adjustment for updated mean HbA1c which led to the conclusion that glycemic exposure correlates very strongly with CVD and mortality, and that this is partly mediated by hyperglycemia-induced renal disease [15]. Steno-2 study, including T2DM patients with microalbuminuria, showed that long-term intensified therapy reduced the risk of $\mathrm{CV}$ events, as well as diabetic nephropathy, DR, and DN [16]. Also, the risk of microalbuminuria has been shown to increase with T2DM duration [17].

Potential associations, of DR and DN with CV outcomes, whether causal or only predictive, have received less attention. We therefore investigated the association of DR and $\mathrm{DN}$ with increased risk of recurrent $\mathrm{CV}$ events, in patients with a recent acute coronary syndrome (ACS).

\section{Materials and Methods}

2.1. Study Design and Patients. The ELIXA (Evaluation of Lixisenatide in Acute Coronary Syndrome) was a randomized, double-blind, placebo-controlled trial designed to assess the effects of lixisenatide added to current T2DM therapy on $\mathrm{CV}$ morbidity and mortality in 6068 patients with a recent ACS.

We examined the primary composite (CV death, nonfatal MI, stroke, or hospitalization for unstable angina), $\mathrm{CV}$ composite (CV death, nonfatal MI, stroke, and hospitalization for heart failure (HF)), each of its components, and all-cause mortality. Details of the trial design, entry criteria, and the main results have been reported previously $[18,19]$.

For this post hoc analysis, all 6068 ELIXA participants were included. Self-reported historical data on DR and DN were collected at screening. Patients were asked to answer "yes," "no," or "unknown" on the presence of DR and/or DN. If DR was present, date of diagnosis was recorded, as well as information on photocoagulation and vitrectomy. However, these interventions were not analyzed further due to a small number of events. Presence of DN was defined as a report of either sensory/motor or autonomic neuropathy. Only "yes" responses were used to define the exposure variables for all subsequent analyses. Blood samples included in this analysis were done at screening by a central laboratory. Duration of T2DM was evaluated based on medical record review or self-report at the screening visit.

\subsection{Statistical Analysis. Baseline characteristics of patients} were stratified by the presence of DR and/or DN. Descriptive data are presented as the mean \pm standard deviation for normally distributed variables and as median (25-75th percentile) for nonnormally distributed variables. Categorical variables are expressed as proportions and were compared by the chi-square test, while continuous variables were compared using $t$-tests or Wilcoxon rank-sum tests, as appropriate. Two proportional hazards regression models were used to assess the association between DR, DN, and recurrent $\mathrm{CV}$ events. Multivariable proportional hazards models were used to assess the association between DR, $\mathrm{DN}$, and primary composite endpoint, CV composite endpoint, components, and all-cause mortality. Both DR and DN were included in the first model and adjusted for the duration of T2DM. In the second model, demographic characteristics and $\mathrm{CV}$ risk factors (age, sex, race, body mass index (BMI), baseline HbA1c, smoking, history of hypertension (HT), heart rate, total cholesterol, low-density lipoprotein (LDL) cholesterol, and triglycerides) were added to the previous model. Both models were adjusted for randomized study treatment. Predictors of DR and DN were determined from multivariable logistic regression model using forward stepwise selection including all variables in Table 1. Twosided $p$ values $<0.05$ were considered significant. No adjustment was made for multiple comparisons. Analyses were performed using the Stata version 13.1 (StataCorp., College Station, TX, USA). 
TABLE 1: Characteristics of all patients at baseline according to the presence of retinopathy and/or neuropathy.

\begin{tabular}{|c|c|c|c|c|}
\hline Characteristic & All patients $n=6068$ & $\begin{array}{c}\text { No retinopathy/ } \\
\text { neuropathy } n=4705\end{array}$ & $\begin{array}{c}\text { Retinopathy and/ } \\
\text { or neuropathy } n=1363\end{array}$ & $p$ value \\
\hline Age (years) & $60.3 \pm 9.7$ & $59.7 \pm 9.7$ & $62.3 \pm 9.2$ & $<0.001$ \\
\hline Male sex $(n(\%))$ & $4207(69.3)$ & $3379(71.8)$ & $828(60.7)$ & $<0.001$ \\
\hline Body weight (kg) & $84.9 \pm 19.4$ & $84.1 \pm 18.9$ & $87.6 \pm 20.9$ & $<0.001$ \\
\hline Body mass index $\left(\mathrm{kg} / \mathrm{m}^{2}\right)$ & $30.2 \pm 5.7$ & $29.9 \pm 5.6$ & $31.2 \pm 6.0$ & $<0.001$ \\
\hline Duration of T2DM (years) & $7.4(2.8,13.6)$ & $6.0(2.0,11.7)$ & $12.4(7.0,20.2)$ & $<0.001$ \\
\hline Categories of T2DM duration $(n(\%))$ & & & & $<0.001$ \\
\hline$\leq 1$ year & $964(15.9)$ & $914(19.4)$ & $50(3.7)$ & \\
\hline$>1-\leq 5$ years & $1294(21.3)$ & $1130(24.0)$ & $164(12.0)$ & \\
\hline$>5-\leq 10$ years & $1359(22.4)$ & $1076(22.9)$ & $283(20.8)$ & \\
\hline$>10$ years & $2451(40.4)$ & $1585(33.7)$ & $866(63.5)$ & \\
\hline Race $(n(\%))$ & & & & $<0.001$ \\
\hline White & $4576(75.4)$ & $3471(73.8)$ & $1105(81.1)$ & \\
\hline Black & $221(3.6)$ & $171(3.6)$ & $50(3.7)$ & \\
\hline Asian & $771(12.7)$ & $669(14.2)$ & $102(7.5)$ & \\
\hline Other & $500(8.2)$ & $394(8.4)$ & $106(7.8)$ & \\
\hline Region $(n(\%))$ & & & & $<0.001$ \\
\hline Africa/Near East & $296(4.9)$ & $228(4.8)$ & $68(5.0)$ & \\
\hline Asia Pacific & $703(11.6)$ & $615(13.1)$ & $88(6.5)$ & \\
\hline Eastern Europe & $1587(26.2)$ & $1115(23.7)$ & $472(34.6)$ & \\
\hline North America & $807(13.3)$ & $564(12.0)$ & $243(17.8)$ & \\
\hline South and Central America & $1944(32.0)$ & $1600(34.0)$ & $344(25.2)$ & \\
\hline Western Europe & $731(12.0)$ & $583(12.4)$ & $148(10.9)$ & \\
\hline Smoking status $(n(\%))$ & & & & $<0.001$ \\
\hline Current & $709(11.7)$ & $579(12.3)$ & $130(9.5)$ & \\
\hline Former & $2746(45.3)$ & $2184(46.4)$ & $562(41.2)$ & \\
\hline Never & $2612(43.1)$ & $1941(41.3)$ & $671(49.2)$ & \\
\hline Diastolic blood pressure $(\mathrm{mmHg})$ & $77 \pm 10$ & $77 \pm 10$ & $76.3 \pm 10$ & $<0.001$ \\
\hline Systolic blood pressure $(\mathrm{mmHg})$ & $130 \pm 17$ & $129 \pm 17$ & $131 \pm 17$ & $<0.001$ \\
\hline Heart rate (beats/min) & $70 \pm 10$ & $70 \pm 10$ & $71 \pm 10$ & 0.027 \\
\hline Fasting plasma glucose (mg/dl) & $148.3 \pm 51.6$ & $145.2 \pm 49.1$ & $159.3 \pm 58.1$ & $<0.001$ \\
\hline Glycated hemoglobin (\%) & $7.7 \pm 1.3$ & $7.6 \pm 1.3$ & $8.0 \pm 1.2$ & $<0.001$ \\
\hline Glycated hemoglobin $(\mathrm{mmol} / \mathrm{mol})$ & $61 \pm 14$ & $60 \pm 14$ & $64 \pm 13$ & $<0.001$ \\
\hline Total cholesterol (mg/dl) & $153.5 \pm 44.6$ & $151.3 \pm 43.5$ & $161.1 \pm 47.3$ & $<0.001$ \\
\hline HDL cholesterol (mg/dl) & $42.9 \pm 10.9$ & $42.6 \pm 10.6$ & $44.1 \pm 11.7$ & $<0.001$ \\
\hline LDL cholesterol (mg/dl) & $78.5 \pm 35.3$ & $77.1 \pm 34.7$ & $83.4 \pm 36.9$ & $<0.001$ \\
\hline Triglycerides (mg/dl) & $137.2(99.1,195.6)$ & $136.3(100.0,192.9)$ & $141.6(99.1,208.0)$ & 0.021 \\
\hline $\mathrm{eGFR}\left(\mathrm{ml} / \mathrm{min} / 1.73 \mathrm{~m}^{2}\right)$ & $76 \pm 21$ & $77 \pm 21$ & $71 \pm 22$ & $<0.001$ \\
\hline Albuminuria $(n(\%))$ & & & & $<0.001$ \\
\hline$<30 \mathrm{mg} / \mathrm{g}$ & $4441(74.3)$ & $3579(77.2)$ & $862(64.2)$ & \\
\hline$\geq 30-<300 \mathrm{mg} / \mathrm{g}$ & $1148(19.2)$ & $819(17.7)$ & $329(24.5)$ & \\
\hline$\geq 300 \mathrm{mg} / \mathrm{g}$ & $389(6.5)$ & $237(5.1)$ & $152(11.3)$ & \\
\hline \multicolumn{5}{|l|}{ Medical history at randomization $(n(\%))$} \\
\hline Hypertension & $4635(76.4)$ & $3463(73.6)$ & $1172(86)$ & $<0.001$ \\
\hline Heart failure & $1358(22.4)$ & $923(19.6)$ & $435(31.9)$ & $<0.001$ \\
\hline Stroke & $331(5.5)$ & $204(4.3)$ & $127(9.3)$ & $<0.001$ \\
\hline Peripheral arterial disease & $393(6.5)$ & $227(4.8)$ & $166(12.2)$ & $<0.001$ \\
\hline Atrial fibrillation & $366(6.0)$ & $247(5.2)$ & $119(8.7)$ & $<0.001$ \\
\hline Percutaneous coronary intervention & $4079(67.2)$ & $3263(69.4)$ & $816(59.9)$ & $<0.001$ \\
\hline Coronary artery bypass grafting & $507(8.4)$ & $363(7.7)$ & $144(10.6)$ & $<0.001$ \\
\hline
\end{tabular}


TABLE 1: Continued.

\begin{tabular}{|c|c|c|c|c|}
\hline Characteristic & All patients $n=6068$ & $\begin{array}{c}\text { No retinopathy/ } \\
\text { neuropathy } n=4705\end{array}$ & $\begin{array}{c}\text { Retinopathy and/ } \\
\text { or neuropathy } n=1363\end{array}$ & $p$ value \\
\hline Qualifying ACS event $(n(\%))$ & & & & $<0.001$ \\
\hline STEMI & $2666(44.0)$ & $2187(46.5)$ & $479(35.2)$ & \\
\hline NSTEMI & $2348(38.7)$ & $1817(38.6)$ & $531(39.0)$ & \\
\hline Unstable angina & $1042(17.2)$ & $693(14.7)$ & $349(25.6)$ & \\
\hline Missing & $9(0.1)$ & $6(0.1)$ & $3(0.2)$ & \\
\hline \multicolumn{5}{|l|}{ Antihyperglycemic therapy $(n(\%))$} \\
\hline Metformin & $4243(69.9)$ & $3367(71.6)$ & $876(64.3)$ & $<0.001$ \\
\hline Sulfonylureas & $2266(37.3)$ & $1779(37.8)$ & 487 (35.7) & 0.16 \\
\hline Insulin & $2891(47.6)$ & $1948(41.4)$ & $943(69.2)$ & $<0.001$ \\
\hline Thiazolidinediones & $128(2.1)$ & $92(2.0)$ & $36(2.6)$ & 0.12 \\
\hline Alpha-glucose inhibitor & $181(3.0)$ & $150(3.2)$ & $31(2.3)$ & 0.08 \\
\hline Dipeptidyl peptidase 4 inhibitor & $226(3.7)$ & $176(3.7)$ & $50(3.7)$ & 0.90 \\
\hline Other & $485(8.0)$ & $384(8.2)$ & $101(7.4)$ & 0.37 \\
\hline
\end{tabular}

Data is presented as means \pm SD, median (25-75th percentile), or percentages. HDL: high-density lipoprotein; LDL: low-density lipoprotein; MI: myocardial infarction; ACS: acute coronary syndrome; eGFR: estimated glomerular filtration rate.

\section{Results}

3.1. Baseline Characteristics. Demographic and clinical characteristics of the 6068 patients are shown in Table 1. In the whole population, the mean age was 60.3 years and known T2DM duration was 7.4 years. However, the reported duration of T2DM varied widely, with $15.9 \%$ of participants having known T2DM for less than 1 year, $21.3 \% \leq 5$ years, $22.4 \%>1-\leq 5$ years, and $40.4 \%$ longer than 10 years (Supplementary Figure 1). Of the whole population, 1363 (22.5\%) reported DR and/or DN. DR was reported in $651(10.7 \%)$ patients, DN in $1060(17.5 \%)$ patients, and both in $348(5.7 \%)$ patients (Figure 1). Of 651 who reported DR, 159 patients $(24.4 \%)$ had prior photocoagulation, and 32 patients (4.9\%) had vitrectomy.

Patients who had DR and/or DN were significantly older (mean 62.3 vs. 59.7 years) and had longer known duration of T2DM (mean 12.4 vs. 6.0 years) than those with neither complication. Smaller proportions of patients with DR and/or DN reported shorter T2DM duration of $<1$ year $(3.7 \%$ vs $19.4 \%)$ and $\leq 5$ years ( $12.0 \%$ vs. $24.0 \%$ ). The distribution of T2DM duration in all patients, as well as in those with and without retinopathy and/or neuropathy, is shown in Figure 2. Participants with DR and/or DN also had higher BMI, were also more likely to be nonsmokers, and had significantly higher glycated hemoglobin, fasting plasma glucose, and total and LDL cholesterol. The subgroup with $\mathrm{DR}$ and/or $\mathrm{DN}$ also had more evidence of renal disease reflected as increased albumin-to-creatinine ratio in comparison with those without $\mathrm{DR}$ and/or DN. Also, they were more likely to have history of CV disease (HT, HF, stroke, peripheral arterial disease, and atrial fibrillation). Finally, patients with $\mathrm{DR}$ and/or DN used more frequently metformin and insulin, while other glucose-lowering agents were similarly distributed among groups (Table 1).

3.2. Retinopathy and/or Neuropathy and CV Outcomes. In univariate analysis, DR was significantly associated with

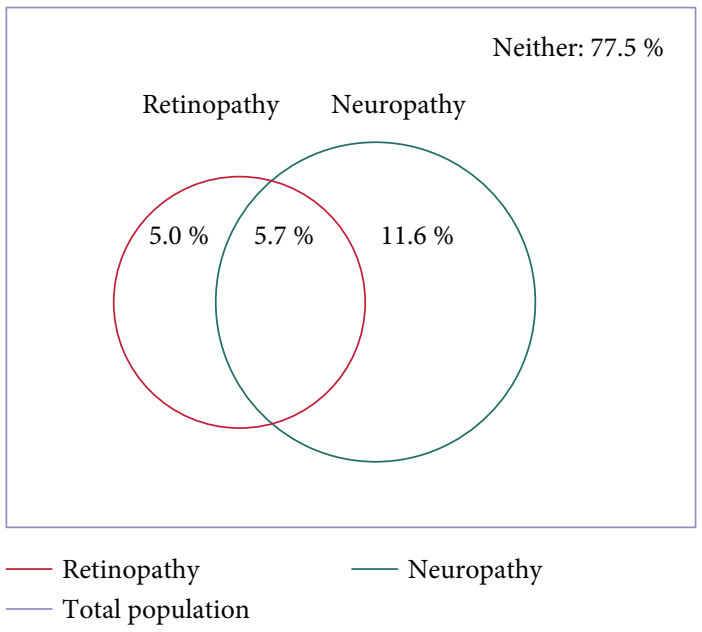

FIgURE 1: Prevalence of retinopathy and/or neuropathy.

primary and $\mathrm{CV}$ composite endpoint, all-cause and $\mathrm{CV}$ death, and all CV events except stroke $(p=0.068)$, while $\mathrm{DN}$ was associated with primary and CV composite endpoint, all-cause death, and all nonfatal CV events, but not CV death $(p=0.08$, Table 2$)$. When both DR and DN were included in the same model, along with T2DM duration, there were no independent associations of either with any of the outcomes, while duration of T2DM remained highly significantly associated with all outcomes (Table 2). Furthermore, the addition of demographic characteristics and CV risk factors to the previous model resulted in neither DR nor DN being associated to any of the outcomes, but identified duration of T2DM as an independent predictor of $\mathrm{CV}$ events, beyond DR, DN, and CV risk factors (Table 2). There were no significant interactions between $\mathrm{DR}$ and $\mathrm{DN}$ in adjusted and unadjusted models. It has previously been reported that no significant interactions were detected with respect to prespecified patient subgroups for the ELIXA 

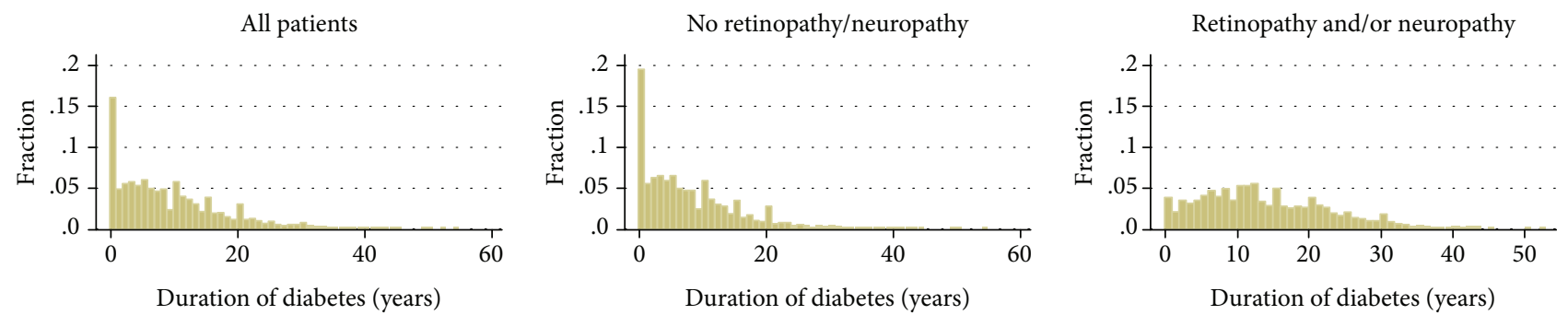

FIGURE 2: Distribution of T2DM duration in all patients, patients with no retinopathy/neuropathy and patients with retinopathy and/or neuropathy.

primary composite outcome, which included T2DM duration $<10$ vs. $>10$ years [19]. In addition, no statistically significant interactions were found with respect to $\mathrm{DR}, \mathrm{DN}$, or duration of T2DM as a continuous variable.

3.3. Predictors of Retinopathy and/or Neuropathy. In both univariate and multivariate analysis, the most significant predictors of both DR and DN were duration of T2DM and insulin use (Table 3). The relationships between presence of DR and DN with duration of T2DM are shown in Supplementary Figures 2a-b. In multivariate analysis, other highly statistically significant $(p<0.001)$ predictors of DR were history of HF and stroke (Table 3 ) and of DN higher weight were previous percutaneous coronary intervention (PCI), total cholesterol, history of peripheral arterial disease, and previous stroke (Table 3). These models were effectively able to discriminate between patients with and without DR (area under the curve (AUC) 0.81) and DN (AUC 0.76).

\section{Discussion}

In this post hoc analysis of a large population selected for having a recent ACS event, together with T2DM, less than one-fourth of patients reported a history of DR, DN, or both. This was a smaller proportion than found in other studies of CV outcomes in T2DM, in which many patients had CV risk factors but not necessarily a completed event. For example, the prevalence of DR in the Trial to Reduce Cardiovascular Events with Aranesp Therapy (TREAT) study-which also relied on patient self-reports-was almost three times higher in a more severely compromised patient cohort with T2DM, chronic kidney disease, and anemia, but also twice as long T2DM duration [20]. The widely varying duration of T2DM in the ELIXA, and the inclusion of a large fraction with very short duration of T2DM, allowed our analysis to examine the relationships between duration of T2DM, presence of DR or DN, and risk of subsequent CV events.

The variability of previously reported associations between DR and/or DN and subsequent CV events could stem from variable T2DM duration and comorbidities of analyzed patient cohorts. The positive association of DR and CV events was observed in various patient cohorts [21-23]. In a recently published study with a large cohort of T2DM patients without the history of CV diseases, the risk of developing CV death, nonfatal MI, or stroke was about $40 \%$ higher in patients with DR and DN [24]. In the meta-analysis including 11,505 patients, DR was associated with 1.7 -fold increased risk for $\mathrm{CV}$ events [25]. However, in the TREAT study, enrolling 4038 patients with T2DM, chronic kidney disease, and anemia, DR was not independently associated with a higher risk of renal or CV morbidity or death, possibly due to longer duration of T2DM and more comorbidities [20].

Our findings on DN are partially in accordance with previously published studies that revealed an association between autonomic DN and CV events [26]. Other studies have shown that DN was associated with a twofold risk increase for peripheral vascular disease [27]. In our study, we investigated the association of both $\mathrm{DN}$ and outcomes, but not peripheral vascular disease. Therefore, our findings on the large cohort of T2DM patients with recent ACS provide novel insight into the association between DN and recurrent $\mathrm{CV}$ events. These findings indicate the importance of the duration of T2DM as a traditional risk factor [28].

Consistent with prior reports, our analysis found that DR and/or DN were associated at baseline with longer T2DM duration, worse glycemic control, more insulin use, and history of prior manifest CV disease. Furthermore, both DR and $\mathrm{DN}$ were associated with increased risk of recurrent $\mathrm{CV}$ events, especially HF hospitalization. However, these associations were no longer statistically significant after adjustment for duration of T2DM, which remained a strong independent predictor of CV events $[20,29,30]$. Thus, DR and DN as well as duration of T2DM appear to be predictors of increased CV risk but, unlike diabetic nephropathy, not themselves are contributors to this risk.

While other relevant studies also adjusted clinical outcomes for the duration of T2DM-among other demographic characteristics and CV risk factors-none of them adjusted for only the known duration of the disease, which makes our findings unique. In a high-risk T2DM patient cohort used in the Veterans Affairs Diabetes Trial (VADT) (1791 subjects) [31], the duration of T2DM was significantly related to $\mathrm{CV}$ events. However, to the best of our knowledge, no studies included similar patient cohort, T2DM patients with recent ACS.

There are several possible explanations for the duration of T2DM being a strong independent predictor of recurrent $\mathrm{CV}$ events in our analysis. Longer duration of the disease may have a direct effect on progression of atherosclerotic lesions, increasing the risk of a recurrent CV event. In addition, it might be associated with autonomic $\mathrm{DN}$ and reduced heart rate variability, increasing the risk of $\mathrm{CV}$ death, which was not the case in our cohort. A long-term increase in 


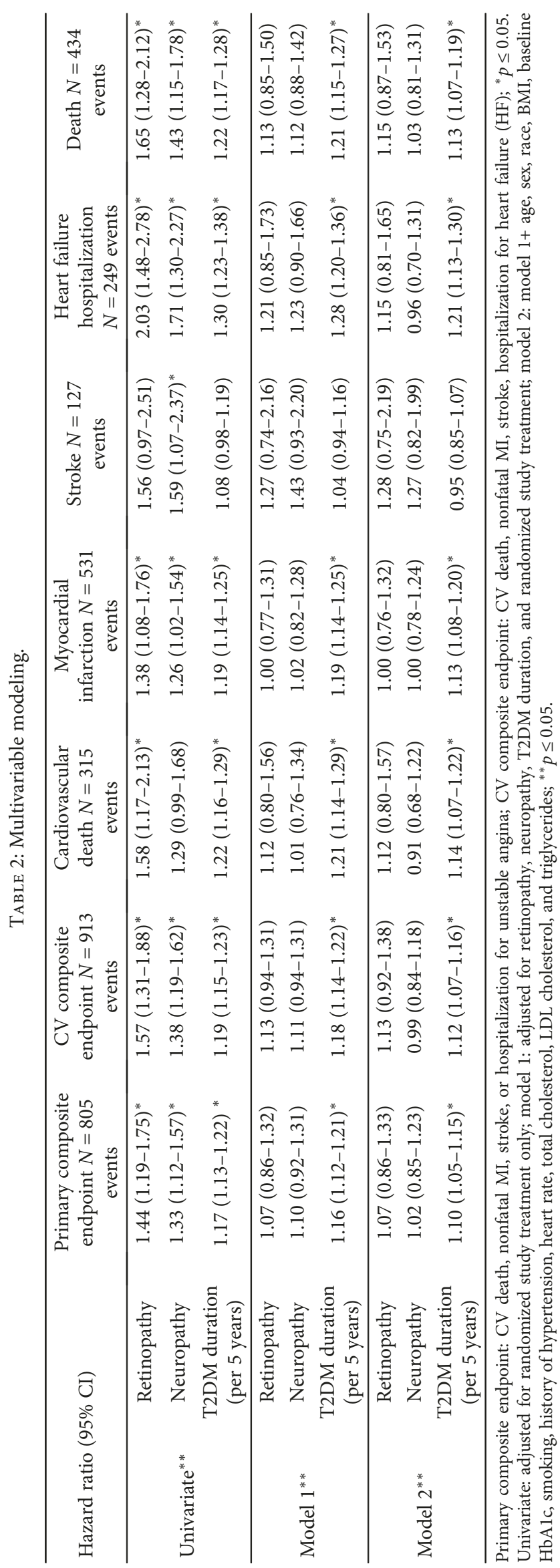


TABLE 3: Predictors of retinopathy and neuropathy multivariate models.

\begin{tabular}{|c|c|c|c|c|}
\hline \multirow{2}{*}{ Parameter } & \multicolumn{2}{|c|}{ Retinopathy } & \multicolumn{2}{|c|}{ Neuropathy } \\
\hline & OR $(95 \% \mathrm{CI})$ & $|Z|$ score & OR $(95 \% \mathrm{CI})$ & $|Z|$ score \\
\hline Duration of diabetes (per 5 years) & $1.48(1.41-1.56)$ & 14.64 & $1.31(1.25-1.37)$ & 11.52 \\
\hline Insulin use & $2.77(2.23-3.46)$ & 9.13 & $2.19(1.86-2.58)$ & 9.36 \\
\hline Weight (per 5 kg) & - & - & $1.09(1.07-1.11)$ & 8.83 \\
\hline Previous PCI & $0.75(0.61-0.91)$ & 2.94 & $0.60(0.51-0.70)$ & 6.32 \\
\hline Total cholesterol (per 10 mg/dl) & - & - & $1.05(1.04-1.07)$ & 6.23 \\
\hline History of PAD & - & - & $1.88(1.45-2.43)$ & 4.82 \\
\hline History of HF & $1.65(1.35-2.02)$ & 4.82 & $1.32(1.12-1.56)$ & 3.23 \\
\hline Previous stroke & $2.06(1.51-2.82)$ & 4.54 & $1.86(1.42-2.45)$ & 4.45 \\
\hline Diastolic blood pressure (per $10 \mathrm{mmHg}$ ) & - & - & $0.88(0.82-0.95)$ & 3.25 \\
\hline Sulphonylurea use & - & - & $1.29(1.11-1.51)$ & 3.24 \\
\hline Previous CABG & $0.72(0.52-0.98)$ & 2.11 & $0.66(0.51-0.86)$ & 3.06 \\
\hline Presence of hypertension & $1.46(1.11-1.91)$ & 2.69 & $1.39(1.12-1.72)$ & 3.00 \\
\hline Body mass index (per $5 \mathrm{~kg} / \mathrm{m}^{2}$ ) & $1.12(1.04-1.22)$ & 2.99 & - & - \\
\hline Glucose (per 10 mg/dl) & $1.02(1.0-1.04)$ & 2.29 & $1.02(1.01-1.03)$ & 2.87 \\
\hline eGFR (per $10 \mathrm{ml} / \mathrm{min} / 1.73 \mathrm{~m}^{2}$ ) & $0.94(0.90-0.98)$ & 2.66 & - & - \\
\hline Male sex & - & - & $1.25(1.06-1.47)$ & 2.60 \\
\hline HDL cholesterol (per 10 mg/dl) & $1.11(1.02-1.20)$ & 2.53 & - & - \\
\hline Age (per 5 years) & - & - & $1.05(1.01-1.10)$ & 2.20 \\
\hline HbAlc (per 1\%) & $1.09(1.00-1.19)$ & 1.98 & - & - \\
\hline
\end{tabular}

Model AUC $=0.81$ (retinopathy) and model AUC $=0.76$ (neuropathy). HF: heart failure; PCI: percutaneous coronary intervention; eGFR: estimated glomerular filtration rate; HDL: high-density lipoprotein; CABG: coronary artery bypass grafting; HbAlc: glycated hemoglobin; PAD: peripheral artery disease.

oxidative stress in T2DM patients may cause increased risk of $\mathrm{CV}$ death. Also, longer exposure to hyperglycemia may simply reflect greater exposure to other, perhaps unmeasured, CV risk factors.

The major limitation of our study was the assessment of DR and DN which was based on patients' answers to self-report questionnaires. Therefore, the prevalence of both might have been underestimated. Such potential misclassification bias might have potentially weakened our results, and, therefore, the real association of DR and $\mathrm{DN}$ with recurrent $\mathrm{CV}$ events could be stronger than presented in this analysis. Another important limitation is the fact that T2DM duration was evaluated based on medical record or self-report and could therefore be underestimated, as patients often remain undiagnosed for many years. Also, having in mind that the study cohort included high-risk patients with T2DM and recent ACS, the results may not be applicable to other populations such as those without $\mathrm{CV}$ disease or with a less advanced stage of it. In addition, the design of this study was cross-sectional and therefore does not provide information on whether or not $\mathrm{DR}$ and $\mathrm{DN}$ developed before or after the onset of any CV disease antecedent to the qualifying ACS event. Finally, our findings should be considered post hoc and hypothesis-generating.

\section{Conclusions}

In a population with recent ACS together with T2DM, recurrent $C V$ events, $\mathrm{DR}$, and $\mathrm{DN}$ were all strongly associated with the T2DM duration. A history of either DR or DN was associated with increased risk of recurrent $\mathrm{CV}$ events. As these associations were eliminated by adjustment for the duration of T2DM, which remained a strong independent predictor of recurrent $\mathrm{CV}$ events, the link between DR and/or DN and these events is not likely to be a causal one. However, history of DR, DN, or both and longer duration of T2DM could be considered clinical markers for high risk of recurrent CV events. It is important to point out that the presence of both DN and DR is expected to positively correlate with patients' level of interaction with healthcare providers. Furthermore, it is expected that their level of healthcare provider interaction would correlate negatively with the frequency and magnitude of adverse outcomes.

\section{Data Availability}

The data used to support the findings of this study are available from the corresponding author upon reasonable request.

\section{Disclosure}

The company, Sanofi, played no role in the design and execution of this analysis.

\section{Conflicts of Interest}

JPS has no competing interests. RBL is a consultant and research support of Sanofi and a research support and spouse/partner of Sanofi, Amgen Inc., and Novartis 
Pharmaceuticals Corporation. $\mathrm{BC}$ has no competing interests. RD is a research support of Sanofi. HCG is an advisory panel of Sanofi, Novo Nordisk Inc., Bristol-Myers Squibb Company, Roche Pharmaceuticals, AstraZeneca Pharmaceuticals LP, GlaxoSmithKline, Bayer HealthCare, LLC, Merck, Boehringer Ingelheim Pharmaceuticals Inc., and Eli Lilly and Company; a consultant of Sanofi; and a research support of Sanofi and Eli Lilly and Company. LVK has a relationship with Sanofi and Novartis Pharmaceuticals Corporation. FL is an employee of Sanofi. EFL is a consultant and spouse/partner of Sanofi; a research support of Amgen Inc., Novartis Pharmaceuticals Corporation, and Sanofi; and a research support and spouse/partner of Sanofi. APM has a relationship with Sanofi, Oxford University, Eli Lilly and Company, and BMS. JMcM has a relationship with GlaxoSmithKline, PPD Development LP, Merck, Oxford University, Amylin Pharmaceuticals Inc., Eli Lilly and Company, Parexel, Bayer HealthCare, LLC, Abbvie, and Sanofi. JLP is an advisory panel of Sanofi, a consultant of Sanofi, and a research support of Sanofi. MCR is a consultant of Eli Lilly and Company, AstraZeneca Pharmaceuticals LP, Sanofi, Valeritas, LLC, and Elcelyx Therapeutics Inc.; a research support of AstraZeneca Pharmaceuticals LP, Eli Lilly and Company, Sanofi, and Novo Nordisk Inc.; and has a relationship with Sanofi. These dualities of interest have been reviewed and managed by Oregon Health \& Science University. SDS is a consultant of Novartis Pharma AG and Amgen Inc. and a research support of Abbott Laboratories Inc., Amgen Inc., DaiichiSankyo Inc., Novartis Pharma AG, Theracos, Boston Scientific, NHLBI, Lone Star Heart, and Sanofi. JCT has no competing interests. MAP is a consultant of Aastrom Biosciences Inc., Abbott Laboratories Inc., Amgen Inc., FibroGen Inc., Cerenis Pharmaceuticals, Concert Pharmaceuticals, GlaxoSmithKline, Hamilton Health Sciences, Medtronic, Merck, Roche Pharmaceuticals, Servier, Teva Pharmaceuticals, and University of Oxford; a research support of Amgen Inc., Celladon, Novartis Pharma AG, and Sanofi; and has a relationship with Novartis Pharma AG.

\section{Authors' Contributions}

The presented analysis was prepared jointly by all authors. The corresponding author and coauthors had full access to the data in the study, contributed to the writing of the manuscript, and had final responsibility for the decision to submit for publication.

\section{Acknowledgments}

The ELIXA trial was supported by Sanofi.

\section{Supplementary Materials}

Supplementary Figure 1. Patient distribution by T2DM duration categories widely varies: $15.9 \%$ of participants have known T2DM for less than 1 year, $21.3 \% \leq 5$ years, $22.4 \%>1-\leq 5$ years, and $40.4 \%$ longer than 10 years. Supplementary Figures 2a-b. Relationship of retinopathy and neuropathy with T2DM duration. (Supplementary Materials)

\section{References}

[1] H. C. Gerstein, "Glucose: a continuous risk factor for cardiovascular disease," Diabetic Medicine, vol. 14, Supplement 3, pp. S25-S31, 1997.

[2] C. S. Fox, L. Sullivan, D'Agostino RB Sr, P. W. Wilson, and Framingham Heart Study, "The significant effect of diabetes duration on coronary heart disease mortality: the Framingham Heart Study," Diabetes Care, vol. 27, no. 3, pp. 704-708, 2004.

[3] for the ADVANCE Collaborative group, S. Zoungas, M. Woodward et al., "Impact of age, age at diagnosis and duration of diabetes on the risk of macrovascular and microvascular complications and death in type 2 diabetes," Diabetologia, vol. 57, no. 12, pp. 2465-2474, 2014.

[4] I. Tkác, "Effect of intensive glycemic control on cardiovascular outcomes and all-cause mortality in type 2 diabetes: overview and metaanalysis of five trials," Diabetes Research and Clinical Practice, vol. 86, Supplement 1, pp. S57-S62, 2009.

[5] T. H. Tung, S. J. Chen, F. L. Lee, J. H. Liu, C. H. Lin, and P. Chou, "A community-based study for the utility values associated with diabetic retinopathy among type 2 diabetics in Kinmen, Taiwan," Diabetes Research and Clinical Practice, vol. 68, no. 3, pp. 265-273, 2005.

[6] S. Tesfaye, N. Chaturvedi, S. E. Eaton et al., "Vascular risk factors and diabetic neuropathy," The New England Journal of Medicine, vol. 352, no. 4, pp. 341-350, 2005.

[7] S. Adler, "Structure-function relationships in diabetic nephropathy: lessons and limitations," Kidney International. Supplement, vol. 60, pp. S42-S45, 1997.

[8] A. I. Adler, E. J. Boyko, J. H. Ahroni, V. Stensel, R. C. Forsberg, and D. G. Smith, "Risk factors for diabetic peripheral sensory neuropathy. Results of the Seattle Prospective Diabetic Foot Study," Diabetes Care, vol. 20, no. 7, pp. 1162-1167, 1997.

[9] B. M. Sörensen, A. J. H. M. Houben, T. T. J. M. Berendschot et al., "Prediabetes and type 2 diabetes are associated with generalized microvascular dysfunction: the Maastricht Study," Circulation, vol. 134, no. 18, pp. 1339-1352, 2016.

[10] I. M. Stratton, A. I. Adler, H. A. Neil et al., "Association of glycaemia with macrovascular and microvascular complications of type 2 diabetes (UKPDS 35): prospective observational study," BMJ, vol. 321, no. 7258, pp. 405-412, 2000.

[11] B. Nazimek-Siewniak, D. K. Moczulski, and W. Grzeszczak, "Risk of macrovascular and microvascular complications in type 2 diabetes: results of longitudinal study design," Journal of Diabetes and its Complications, vol. 16, no. 4, pp. 271-276, 2002.

[12] M. A. Gall, P. Hougaard, K. Borch-Johnsen, and H. H. Parving, "Risk factors for development of incipient and overt diabetic nephropathy in patients with non-insulin dependent diabetes mellitus: prospective, observational study," BMJ, vol. 314, no. 7083, pp. 783-788, 1997.

[13] B. C. Astor, S. I. Hallan, E. R. Miller, E. Yeung, and J. Coresh, "Glomerular filtration rate, albuminuria, and risk of cardiovascular and all-cause mortality in the US population," American Journal of Epidemiology, vol. 167, no. 10, pp. 1226-1234, 2008.

[14] T. Ninomiya, V. Perkovic, B. E. de Galan et al., "Albuminuria and kidney function independently predict cardiovascular and renal outcomes in diabetes," Journal of the American Society of Nephrology, vol. 20, no. 8, pp. 1813-1821, 2009. 
[15] I. H. de Boer, X. Gao, P. A. Cleary et al., "Albuminuria changes and cardiovascular and renal outcomes in type 1 diabetes: the DCCT/EDIC study," Clinical Journal of the American Society of Nephrology, vol. 11, no. 11, pp. 1969-1977, 2016.

[16] P. Gæde, P. Vedel, N. Larsen, G. V. H. Jensen, H.-H. Parving, and O. Pedersen, "Multifactorial intervention and cardiovascular disease in patients with type 2 diabetes," The New England Journal of Medicine, vol. 348, no. 5, pp. 383-393, 2003.

[17] V. Monhart, "Microalbuminuria. From diabetes to cardiovascular risk," Vnitřní Lékařství, vol. 57, pp. 293-298, 2011.

[18] R. Bentley-Lewis, D. Aguilar, M. C. Riddle et al., "Rationale, design, and baseline characteristics in Evaluation of LIXisenatide in Acute Coronary Syndrome, a long-term cardiovascular end point trial of lixisenatide versus placebo," American Heart Journal, vol. 169, no. 5, pp. 631-638.e7, 2015.

[19] M. A. Pfeffer, B. Claggett, R. Diaz et al., "Lixisenatide in patients with type 2 diabetes and acute coronary syndrome," The New England Journal of Medicine, vol. 373, no. 23, pp. 2247-2257, 2015.

[20] N. A. Bello, M. A. Pfeffer, H. Skali et al., "Retinopathy and clinical outcomes in patients with type 2 diabetes mellitus, chronic kidney disease, and anemia," BMJ Open Diabetes Research \& Care, vol. 2, no. 1, article e000011, 2014.

[21] G. Targher, L. Bertolini, L. Zenari et al., "Diabetic retinopathy is associated with an increased incidence of cardiovascular events in type 2 diabetic patients," Diabetic Medicine, vol. 25, no. 1, pp. 45-50, 2008.

[22] R. Klein, A. R. Sharrett, B. E. Klein et al., "The association of atherosclerosis, vascular risk factors, and retinopathy in adults with diabetes: the atherosclerosis risk in communities study," Ophthalmology, vol. 109, no. 7, pp. 1225-1234, 2002.

[23] N. Cheung, S. Rogers, D. J. Couper, R. Klein, A. R. Sharrett, and T. Y. Wong, "Is diabetic retinopathy an independent risk factor for ischemic stroke?," Stroke, vol. 38, no. 2, pp. 398401, 2007.

[24] J. R. W. Brownrigg, C. O. Hughes, D. Burleigh et al., "Microvascular disease and risk of cardiovascular events among individuals with type 2 diabetes: a population-level cohort study," The Lancet Diabetes and Endocrinology, vol. 4, no. 7, pp. 588597, 2016.

[25] R. S. Rosenson, P. Fioretto, and P. M. Dodson, "Does microvascular disease predict macrovascular events in type 2 diabetes?," Atherosclerosis, vol. 218, no. 1, pp. 13-18, 2011.

[26] C. M. Forsblom, T. Sane, P. H. Groop et al., "Risk factors for mortality in type II (non-insulin-dependent) diabetes: evidence of a role for neuropathy and a protective effect of HLA-DR4," Diabetologia, vol. 41, no. 11, pp. 1253-1262, 1998.

[27] L. Kärvestedt, E. Mårtensson, V. Grill et al., "Peripheral sensory neuropathy associates with micro- or macroangiopathy: results from a population based study of type 2 diabetic patients in Sweden," Diabetes Care, vol. 32, no. 2, pp. 317322, 2009.

[28] S. Del Prato, "Megatrials in type 2 diabetes. From excitement to frustration?," Diabetologia, vol. 52, no. 7, pp. 1219-1226, 2009.

[29] M. Lovestam-Adrian, C. Hansson-Lundblad, and O. Torffvit, "Sight-threatening retinopathy is associated with lower mortality in type 2 diabetic subjects: a 10-year observation study," Diabetes Research and Clinical Practice, vol. 77, no. 1, pp. 141147, 2007.
[30] H. H. Parving, E. Hommel, E. Mathiesen et al., "Prevalence of microalbuminuria, arterial hypertension, retinopathy and neuropathy in patients with insulin dependent diabetes," $B M J$, vol. 296, no. 6616, pp. 156-160, 1988.

[31] W. C. Duckworth, C. Abraira, T. E. Moritz et al., "The duration of diabetes affects the response to intensive glucose control in type 2 subjects: the VA Diabetes Trial," Journal of Diabetes and its Complications, vol. 25, no. 6, pp. 355-361, 2011. 


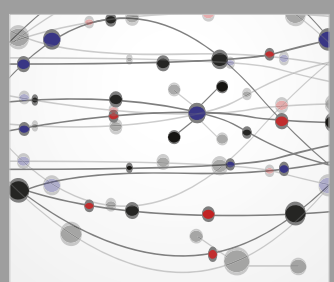

The Scientific World Journal
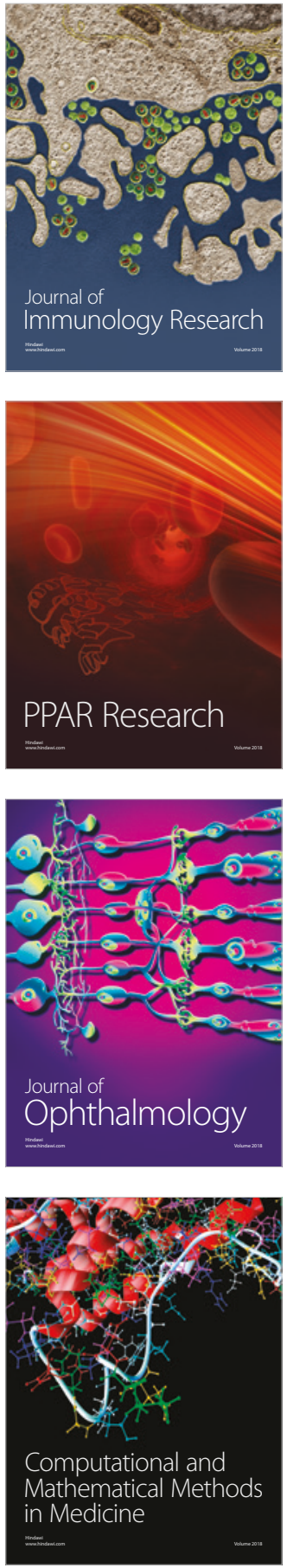

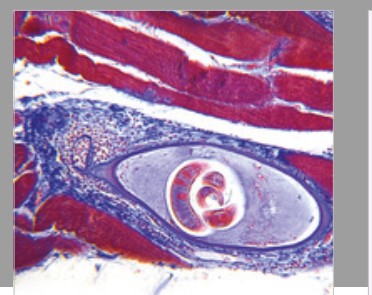

Gastroenterology Research and Practice

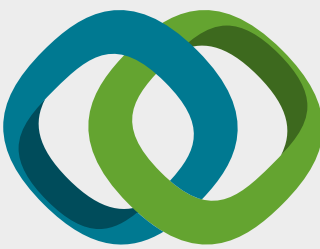

\section{Hindawi}

Submit your manuscripts at

www.hindawi.com
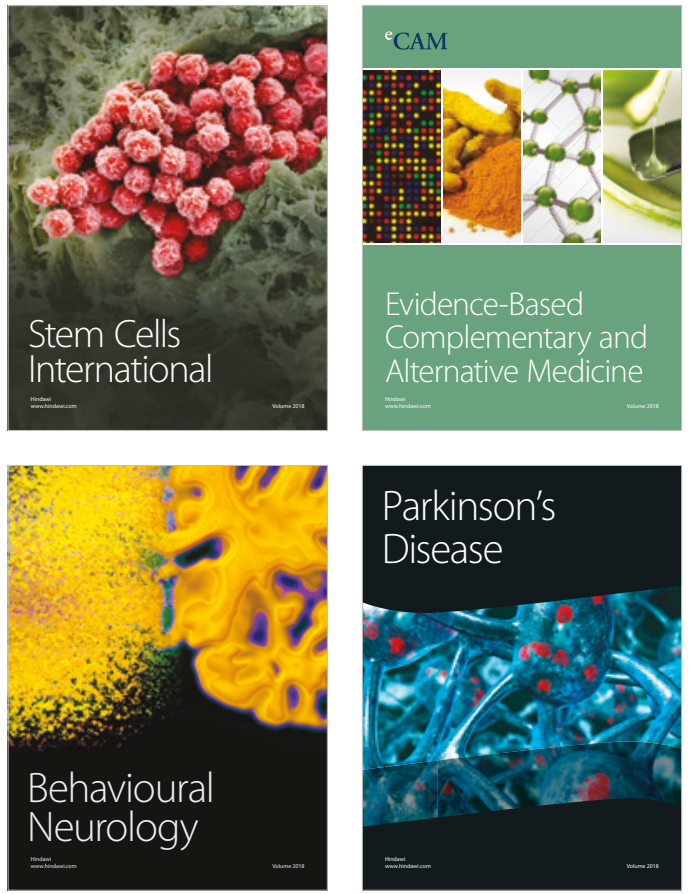

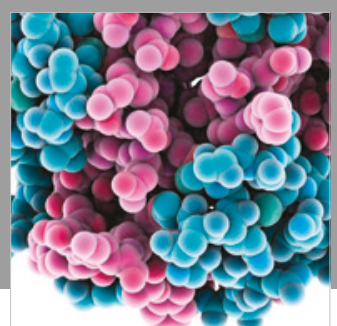

ournal of

Diabetes Research

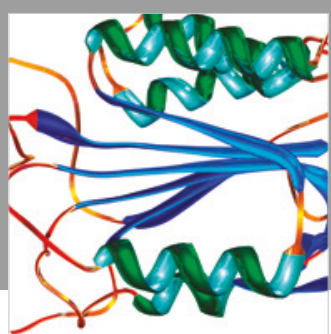

Disease Markers
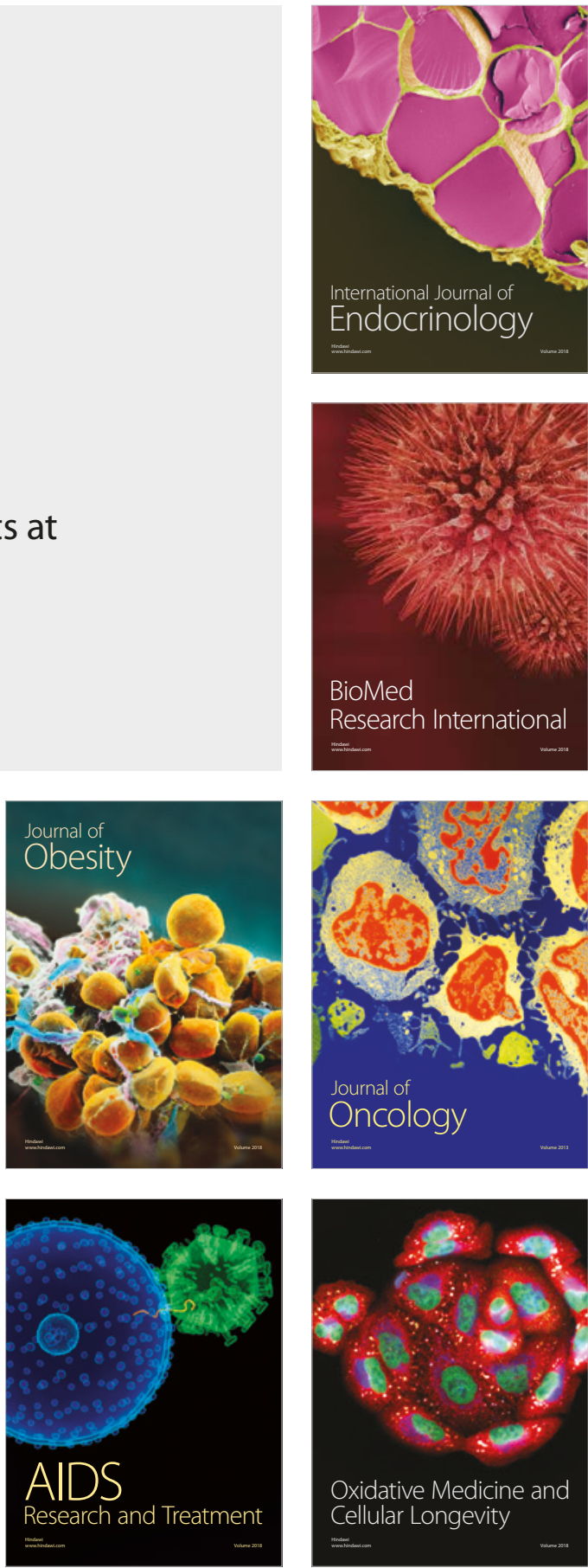